\title{
Nanoparticles influence on dye-sensitized solar cells based on $\mathrm{TiO}_{2}$
}

\author{
Kurapati Srinivas \\ Nanotechnology Group, Department of Physics, GMR Institute of Technology, Rajam, A.P India
}

Email address:

srinkura@gmail.com, srinivas.k@gmrit.org

To cite this article:

Kurapati Srinivas. Nanoparticles Influence on Dye-Sensitized Solar Cells Based on $\mathrm{TiO}_{2}$. American Journal of Nanoscience and Nanotechnology. Vol. 2, No. 3, 2014, pp. 32-39. doi: 10.11648/j.nano.20140203.11

\begin{abstract}
Today, Solar cell technology is in limited use due to the relatively high manufacturing cost of silicon based technology, And the low power efficiency of organic polymer based technology. However, Research is being done on hybrid cells based on dye-sensitizing organic polymers and a thin transparent conducting oxide layer comprised of nanoparticles. These cells could offer the same ease of manufacturing as organic cells, with improved efficiency. Currently, although the improved efficiency is promising, It is still far below silicon based solar cells. The nanotubes clearly show longer response times, Which correlate to longer electron lifetimes, Which means less recombination of electron-hole pairs. This will ultimately translate to higher energy output. This paper will explore the role of nanomaterials in this flexible solar cell technology. A discussion on current efforts to improve efficiency will follow.
\end{abstract}

Keywords: Solar Cell, Dye Sensitized Solar Cell, Nanomaterials, Nanostructures, Efficiency

\section{Introduction}

Today, due to the increasing global demands on energy, it is imperative that a renewable energy source be determined, that is cost effective and reliable. Solar cell technology has shown much promise over the years to replace the use of fossil fuels. However, with the current technology, the cost per watt is rather high due to the high cost of manufacturing silicon-based solar cells. The cost per watt can be lowered two ways. Lower the manufacturing cost, or increase the amount of power output for the same cost. The latter is related to efficiency of the device. In other words, the efficiency is the amount of energy output vs. the amount of energy coming in. In Figure 1, we can see the chronology of the efficiencies for different types of solar cell technology. The current best is around thirty-six percent. Clearly, a more efficient way of converting sunlight into energy needs to be researched in order to make solar cell technology economically viable. Most traditional solar cells rely on a semiconductor (typically silicon) for both light absorption, and charge transport. A fairly new, promising method separates these functions. Organic dyes (dye sensitizers), which are sensitive to light, can absorb a broader range of the sun's spectrum. When a photon hits the dye, an electron in the dye becomes excited and is injected into the conduction band of a nanocrystalline semiconductor oxide, where charge transport takes place. Electrons lost from the dye, are regenerated by a redox electrolyte, usually an inorganic solvent. These components are sandwiched between substrates of transparent conducting oxide. It appears that the enormous surface area per unit volume of nanoparticles can increase the photon to current ratio. Future studies are focusing on controlling the order and shape of the particles to increase the photon to current ratio even further. Although the mechanisms and processes of the dye sensitizer, electrolyte, and conducting substrate are worthy of further study, the focus of this paper will be to review the material properties of $\mathrm{TiO}_{2}$ nanoparticles, and explore the mechanisms that make it a promising material in improving the efficiency of dye sensitized solar cells (DSC).A brief review of the operational theory will be followed by a discussion of the nanocrystalline oxide layer. Photoelectrodes of dye sensitized solar cells (DSSCs) are nanostructured by nature for effectively ferrying electrons and holes [1,2]. The rapid advances of nanomaterials and nanotechnology in recent years are therefore propitious in promoting the development of DSSCs [3]. The nanoscale engineering of new photoelectrode architectures should not only enhance our understanding of the inherent working of DSSCs but also help to improve the cell performance, especially in the emerging frontiers such as flexible DSSCs 
and solid-state DSSCs [4-8]. In a series of recent work, we have demonstrated the viability of new photoanode architectures in DSSCs comprising building blocks of $\mathrm{ZnO}$ nanoplates [9], $\mathrm{ZnO}$ nanotetrapods $[7,10,11]$ and $\mathrm{TiO}_{2}$ nanospindles [12]. This article we reviewed our systemic studies on DSSCs based on $\mathrm{ZnO}$ nanotetrapods related photoanodes, namely, pure $\mathrm{ZnO}$ nanotetrapods based photoanode [7], $\mathrm{ZnO}$ nanotetrapods/ $\mathrm{SnO}_{2}$ nanoparticles composite photoanode with [10] andwithout calcination [11].

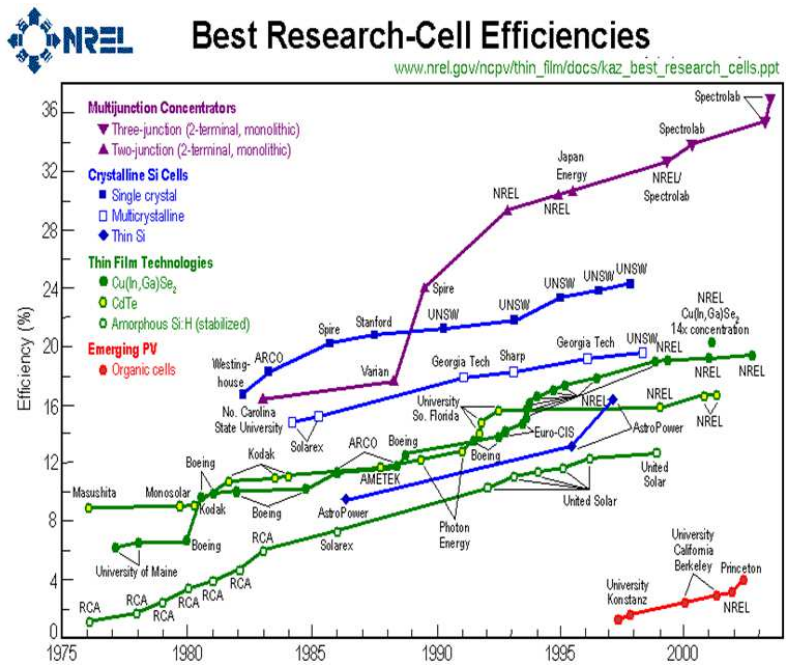

Figure 1. Chronology of solar cell efficiency according to different methodology. [1]

\section{Structure and Mechanism of Dye- Sensitized Solar Cell}

DSC's, shown in Figure 2, rely on processes likened to photosynthesis. In photosynthesis, light is converted into chemical energy. Chlorophyll and other pigments can eject electrons through photo-induced charge separation when struck by photons. The main component of a DSC is a semiconducting material with a wide band gap. One such material is titanium dioxide $\left(\mathrm{TiO}_{2}\right)$. This is deposited as a thin layer onto a transparent conducting oxide (TCO) substrate using a sol-gel technique. The $\mathrm{TiO}_{2}$ layer is also in contact with a monolayer of polymer dye which has commonly been a ruthenium complex, also known as N3 [2].Exposure to sunlight causes electrons in the dye to become excited. This phenomenon is called photoexcitation. The electrons are then ejected from the dye and into the conduction band of the semiconducting oxide layer. Regeneration of the lost

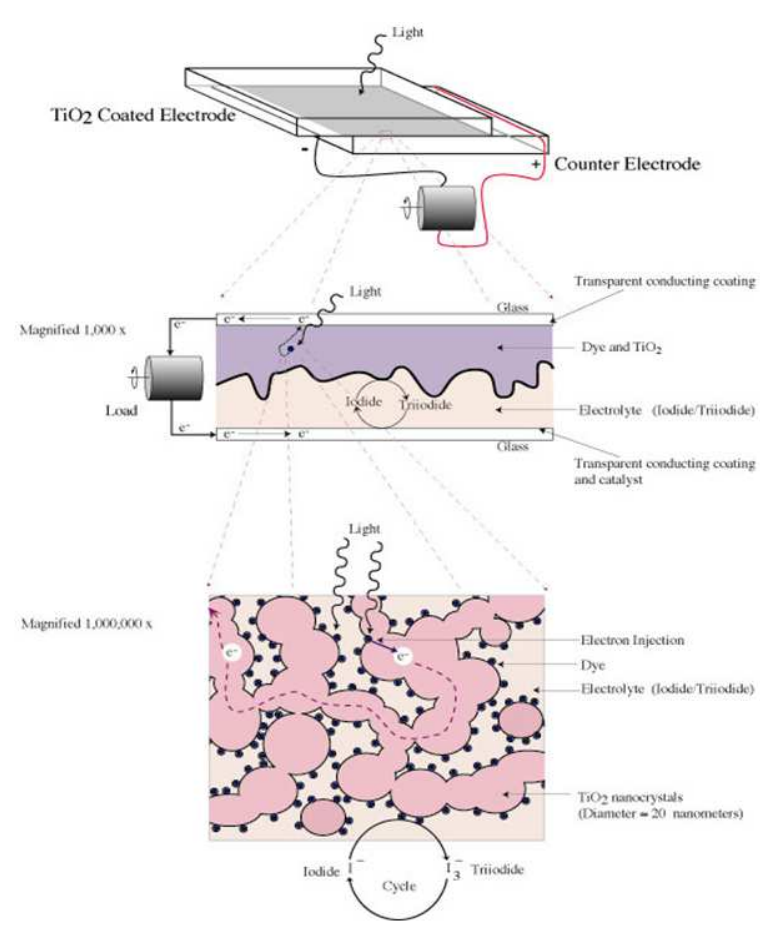

Figure 2. Schematic of a dye sensitized solar cell. [3]

electrons is handled by a redox process within an electrolyte, commonly an iodide/triiodide couple, which is in contact with the dye. The final component is a layer of TCO. The voltage generated is related to the difference between the redox potential of the electrolyte and the Fermi level of the electron within the solid. The electron that was ejected from the dye diffuses through the $\mathrm{TiO}_{2}$ layer and into the conductive oxide electrode where it can be used as current

In recent years, the concept of utilizing nanomaterialbased architectures in light energy conversion devices has emerged as an alternative to single crystalline based photovoltaic devices, dye sensitized solar cell (DSSC) has attracted the attention of many researchers for its low energy cost and low pollution to environment. Figure 2 shows a schematic of the interior of a DSSC showing the principle of how the device operates. The typical configuration is as follows: the heart of the device is the mesoporous oxide layer composed of a network of $\mathrm{TiO}_{2}$ nanostructures that have been sintered together to establish electronic conduction. Typically, the film thickness is 10 to $20 \mu \mathrm{m}$ and the nanoparticle is $10-30 \mathrm{~nm}$ in diameter. The porosity of the film is about $50-60 \%$. The mesoporous layer is deposited on a transparent conducting oxide(TCO) on a glass or plastic substrate 


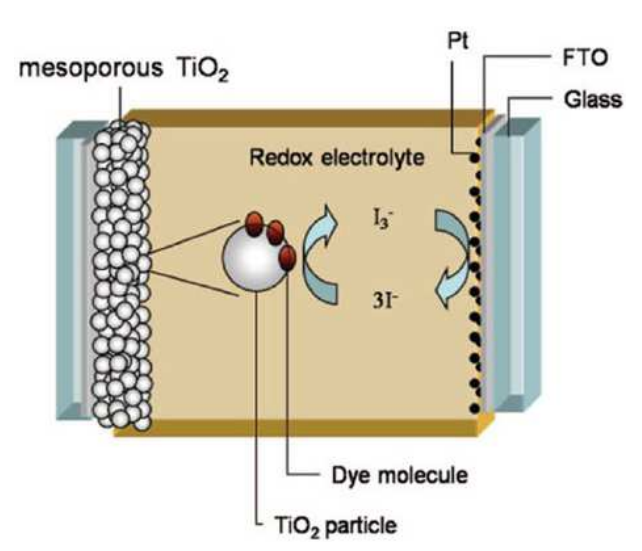

Figure 3. Schematic overview of a dye-sensitized solar cell.

Attached to the surface of the nanocrystalline film is a monolayer of the charge-transfer dye. One of the most important dye is Cisdiisothiocyanato- bis(2,2'-bipyridyl4,4'-dicarboxylato) ruthenium(II) bis(tetrabutylammonium) (N719), as shown in Figure 4. The nanoporous structure of the $\mathrm{TiO}_{2}$ film provides high internal surface area to accommodate sufficient amount of dye for efficient light absorption. It also ensures that each dye molecule is in direct contact with (cathode) is a similar TCO glass substrate but is coated with platinum catalyst particles, The electrodes are attached together with a thermoplastic polymer film that functions both as an edge sealant and spacer forming a sandwich-like thin layer electrochemical cell where an electrolyte layer fills the gap between the electrodes.

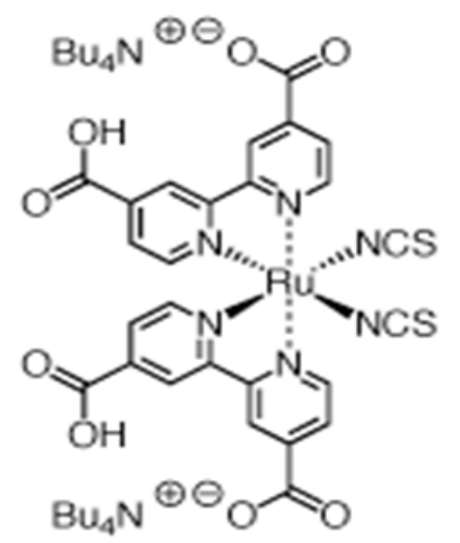

Figure 4. Chemical structure of N719 dye.

The nanoporous structure of the $\mathrm{TiO}_{2}$ film provides high internal surface area to accommodate sufficient amount of dye for efficient light absorption. It also ensures that each dye molecule is in direct contact with (cathode) is a similar TCO glass substrate but is coated with platinum catalyst particles, The electrodes are attached together with a thermoplastic polymer film that functions both as an edge sealant and spacer forming a sandwich-like thin layer electrochemical cell where an electrolyte layer fills the gap between the electrodes The operating principle of DSC is summarized in Figure.5.

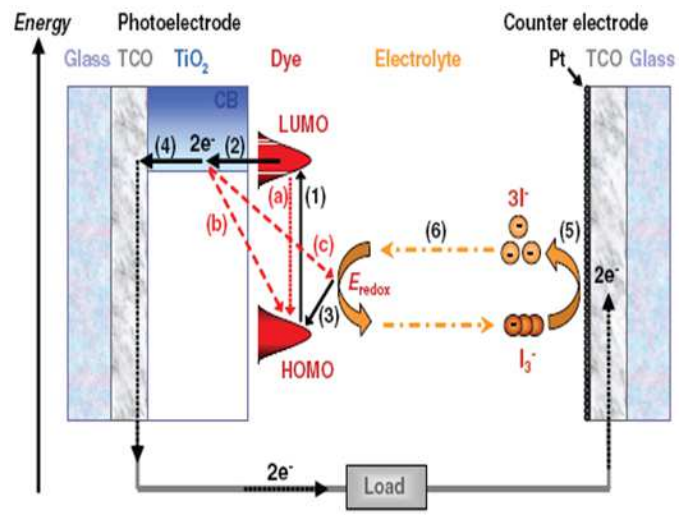

Figure 5. Operating principle of the dye solar cell, showing the forward and backward reactions.

When the dye absorbs a photon an electron is excited from a low-energy state (highest occupied molecular orbital, HOMO) to a high-energy state (lowest unoccupied molecular orbital, LUMO) of the molecule (1). This is followed by electron injection to the $\mathrm{TiO}_{2}$ conduction band (2), and the oxidized dye is regenerated by electron capture from the redox electrolyte (3). The injected electron travels by diffusion in the $\mathrm{TiO}_{2}$ film until it finds its way to the substrate contact where it is released to the external electrical circuit (4). The electron is returned to the cell via an electrolyte reduction reaction at the counter electrode (5). The electrical circuit of the cell is completed by ionic transport of the redox pair in the electrolyte (6). The main back-reactions limiting the photocurrent are indicated with red arrows: (a) radiationless relaxation of the excited state of the dye, (b) recombination of the electrons with the oxidized dye, (c) and with the tri-iodide in the electrolyte.

$$
\begin{aligned}
& \mid \begin{array}{c}
\text { Photoelectrode } \\
\mathrm{S}+h v \rightarrow \mathrm{S}^{*} \quad \text { light absorption by the dye } \\
\mathrm{S}^{*} \rightarrow \mathrm{S}^{+}+\mathrm{e}^{-}\left(\mathrm{TiO}_{2}\right) \quad \text { electron injection }
\end{array} \\
& \begin{array}{|l|}
2 \mathrm{~S}^{+}+3 \mathrm{I}^{-}(\mathrm{PE}) \rightarrow 2 \mathrm{~S}+\mathrm{I}_{3}^{-}(\mathrm{PE}) \quad \text { dye regeneration } \\
\mathrm{e}^{-}\left(\mathrm{TiO}_{2}\right) \rightarrow \mathrm{e}^{-}(\mathrm{PE}) \quad \text { electron transport } \\
\hline \begin{array}{l}
\text { Counter electrode } \\
\mathrm{I}_{3}^{-}(\mathrm{CE})+2 \mathrm{e}^{-}(\mathrm{CE}) \rightarrow 3 \mathrm{I}^{-}(\mathrm{CE}) \\
\text { overall charge transfer reaction }
\end{array} \\
\begin{array}{l}
\text { Electrolyte } \\
3 \mathrm{I}^{-}(\mathrm{CE}) \rightarrow 3 \mathrm{I}^{-}(\mathrm{PE}) \quad \text { Iodide and tri-iodide diffusion } \\
\mathrm{I}_{3}^{-}(\mathrm{PE}) \rightarrow \mathrm{I}_{3}^{-}(\mathrm{CE})
\end{array}
\end{array} \\
& \text { Total reactions: } \\
& 3 \mathrm{I}^{-}(\mathrm{PE})+2 h v \rightarrow \mathrm{I}_{3}^{-}(\mathrm{PE})+2 \mathrm{e}^{-}\left(\mathrm{TiO}_{2}\right) \quad \text { Photoelectrode } \\
& \mathrm{e}^{-}(\mathrm{CE})+h v \rightarrow \mathrm{e}^{-}(\mathrm{PE}) \quad \text { Cell }
\end{aligned}
$$

The much simplified picture of the energetics and kinetics for a working DSC device that emerged in the early research is still useful as an introduction of working principles. The chemical complexity of the device must, 
however, be understood and mastered to improve our ability to identify predictive materials and optimized structure/function relationships. With reference to the different reactions in Figure the kinetic data for the different electron transfer processes taking place at the oxide/dye/electrolyte interface for state-of-the-art DSCs are summarized in Figure 5. One of the most astounding findings in DSC research is the ultrafast injection from the excited Ru-complex in the $\mathrm{TiO}_{2}$ conduction band, reaction 2. Although the detailed mechanism of the injection process is still under debate, it is generally accepted that a fast femtosecond component is observed for this type of sensitizer directly attached to an oxide surface For DSC device performance, the time scales of the injection process should be compared with decay of the excited state of the dye to the ground state, reaction 1 . This is given by the excited state lifetime of the dye, which for typical used in DSSCs is 20-60 ns. Interestingly, Durrant and co-workers have observed a much slower electron injection in a complete DSC device with halftimes Recombination processes are indicated by red arrows1. around $150 \mathrm{ps}$. This would then be slow enough for kinetic competition between electron injection and excited state decay of the dye with potential implications for the overall DSC performance19. The interception of the oxidized dye by the electron donor, normally I-, is in the microsecond time domain. For a turnover number, that is, the cycle life of the sensitizer in the DSC device, to be above 108, which is required for a DSC lifetime of 20 years in outdoor conditions, the lifetime of the oxidized dye must be $>100 \mathrm{~s}$ if the regeneration time is $1 \mu \mathrm{s}$. This is achieved by the best-performing Rucomplexes 20 . When the dye-sensitized mesoporous solar cell was first presented, perhaps the most puzzling phenomenon was the highly efficient charge transport through the nanocrystalline $\mathrm{TiO}_{2}$ layer. The mesoporous electrodes are very much different compared with their compact analogues because (i) the inherent conductivity of the film is very low, (ii) the small size of the individual colloidal particles does not support a built-in electrical field, and (iii) the oxide particles and the electrolyte-containing pores form interpenetrating networks whose phase boundaries produce a junction of huge contact area. These films may be viewed as an ensemble of individual particles through which electrons can percolate by hopping from one crystallite to the next. The charge transport mechanisms in DSSC are still under keen debate today. The kinetics of the back-electron-transfer reaction from the conduction band to the oxidized sensitizer follow a multi exponential time law, occurring on a microsecond to millisecond time scale depending on electron concentration in the semiconductor and thus the light intensity. Recombination of electrons in $\mathrm{TiO}_{2}$ with acceptors in the electrolyte is normally referred to as the electron lifetime. Lifetimes observed with the I-/I3- are very long (1-20 ms under one sun light intensity) compared with other redox systems used in DSC, explaining the success of this redox couple. Counter electrodes for DSCs with I-/I3- electrolytes can be rather easily prepared by deposition of a thin catalytic layer of platinum onto a conducting glass substrate.

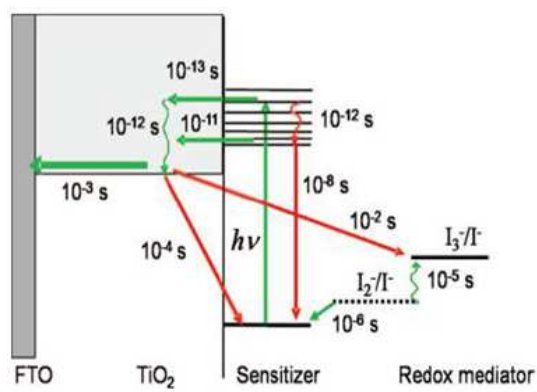

Figure 6. Overview of processes and typical time constants under working Conditions

Best performance and long-term stability has been achieved with nanoscale $\mathrm{Pt}$ clusters. Charge transfer resistances of less than $1 \Omega \mathrm{cm} 2$ can be achieved. In summary, photoexcitation of the latter results in the injection of an electron into the conduction band of the oxide, leaving the dye in its oxidized state. The dye is restored to its ground state by electron transfer from the electrolyte, usually an organic solvent containing the iodide/triiodide redox system. The regeneration of the sensitizer by iodide intercepts the recapture of the conduction band electron by the oxidized dye. The I3- ions formed by oxidation of I- diffuse a short distance $(<50 \mu \mathrm{m})$ through the electrolyte to the cathode, which is coated with a thin layer of platinum catalyst, where the regenerative cycle is completed by electron transfer to reduce I3- to I-.

Some numbers for typical materials and relative concentrations of the different species in the mesoporous system under normal working conditions (1 sun illumination) are noted in the recent paper by O'Regan and Durrant14:

- Under working conditions there are about 10 electrons per $\mathrm{TiO} 2$ particle.

- More than $90 \%$ of electrons in $\mathrm{TiO}_{2}$ are trapped and $<10 \%$ in the conduction band.

- There are $\sim 10000$ adsorption sites for $\mathrm{H}+$ on an 18 nm(diameter) $\mathrm{TiO}_{2}$ particle.

- $\mathrm{A} \mathrm{TiO}_{2}$ particle $(18 \mathrm{~nm})$ has $\sim 600$ dye molecules on the surface.

- Each dye molecule absorbs a photon once per second.

- The flux of electron injection into the $\mathrm{TiO}_{2}$ particle is $\sim 600 \mathrm{~s}-1$.

- Under working conditions, about 1 dye per $150 \mathrm{TiO}_{2}$ particles is in its oxidized state.

- The total volume fraction of the solutes in the electrolyte is $\sim 10-20 \%$.

- In the pore volume around the $\mathrm{TiO}_{2}$ particle, there will be $\sim 1000$ I- and 200 I3-ions.

- The concentration of iodine, $\mathrm{I} 2$, is $<1 \mu \mathrm{M}$, that is, about one free iodine per $10000 \mathrm{TiO}_{2}$ particles.

Figure 7 shows the response time versus open circuit voltage. The plot for 


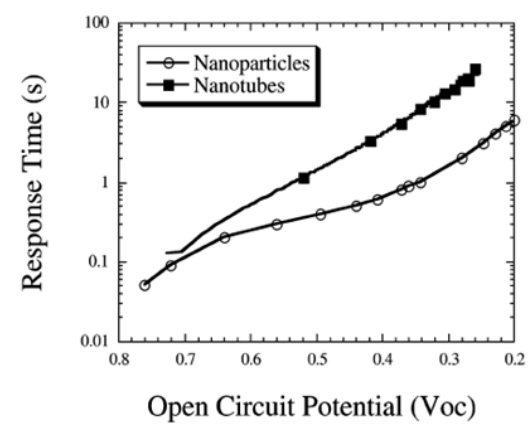

Figure 7. Response time vs. open circuit voltage for nanoparticles and nanotubes. [5]

the nanotubes clearly show longer response times, which correlate to longer electron lifetimes, which means less recombination of electron-hole pairs. This will ultimately translate to higher energy output. These results are very promising, and it is predicted that photocurrent efficiencies could approach the ideal limit of $31 \%$ by lengthening the nanotube length.

\section{Materials of Dye-Sensitized Solar Cell}

The major components of DSSC include dye sensitizers, n-type semiconductor and redox electrolyte. A dye sensitizer is attached to the semiconductor surface by functional anchor groups (usually carboxyl group) to harvest wide spectral distributed light and transfer the energy from photons to excite electrons. If the energy of the exited electron is sufficiently high, it may be injected to the conductive band of the n-type semiconductor through the anchor group and then transported to the photoelectrode. Exited electron injection yields an oxidized sensitizer, which must be reduced by the redox couple in the electrolyte. The injected electrons return to the dark counter electrode through the external circuit where they reduce the oxidized redox couple. To get high power conversion efficient (PCE), high generation rate of photoelectron in the sensitizer, rapid and efficiency electron transport in the semiconductor and fast recovery and diffusion rate of redox couple in the electrolyte are essential.

As one of the crucial parts in dye-sensitized solar cells (DSSCs), the photosensitizer should fulfill some essential characteristics:

(1) The absorption spectrum of the photosensitizer should cover the whole visible region and even the part of the near-infrared (NIR).

(2) The photosensitizer should have anchoring groups ($\mathrm{COOH},-\mathrm{H}_{2} \mathrm{PO}_{3},-\mathrm{SO}_{3} \mathrm{H}$, etc.) to strongly bind the dye onto the semiconductor surface.

(3) The excited state level of the photosensitizer should be higher in energy than the conduction band edge of ntype semiconductor (n-type DSCs), so that an efficient electron transfer process between the excited dye and conduction band (CB) of the semiconductor can take place.
In contrast, for p-type DSCs, the HOMO level of the photosensitizer should be at more positive potential than the valence band $(\mathrm{VB})$ level of p-type semiconductor.

(4) For dye regeneration, the oxidized state level of the photosensitizer must be more positive than the redox potential of electrolyte.

(5) Unfavorable dye aggregation on the semiconductor surface should be avoided through optimization of the molecular structure of the dye or by addition of coadsorbers that prevent aggregation. Dye aggregates can, however, be controlled ( $\mathrm{H}$ - and Jaggregates) leading to an improved performance compared with a monomer dye layer.

(6) The photosensitizer should be photostable, and electrochemical and thermal stability are also required.

\section{Nanostructured Photoelectrodes for Dye-Sensitized Solar Cells}

Nanotechnology opens a door to tailing materials and creating various nanostructures for use in dye-sensitized solar cells. This review classifies the nanostructures into (1) nanoparticles, which offer large surface area to photoelectrode film for dye-adsorption, (2) core-shell structures, which are derived from the nanoparticles however with a consideration to reduce charge recombination by forming a coating layer, (3) onedimensional nanostructures such as nanowires and nanotubes, which provide direct pathways for electron transport much faster than in the nanoparticle films, and (4) three-dimensional nanostructures such as nanotetrapods, branched nanowires or nanotubes, and oxide aggregates, which not only emphasize providing large surface area but also aim at attaining more effective light harvesting and charge transport or collection. The review ends with an outlook proposing that the oxide aggregates are a potentially promising structure which may possibly achieve higher efficiency than the record by reason that the bifunction of aggregates in providing large surface area and generating light scattering allows for photoelectrode film thinner than usual and thus decreases the charge recombination of DSCs.
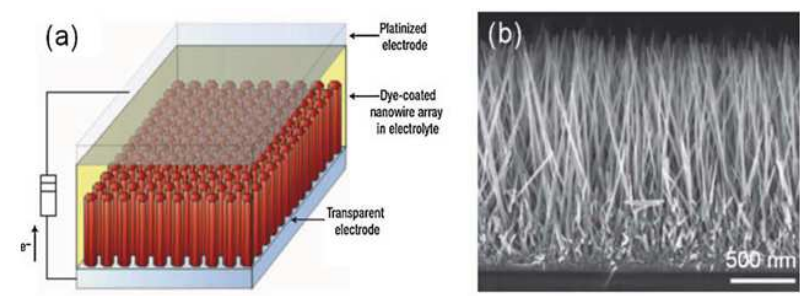

Figure 8. ZnO nanowire dye-sensitized solar cell [66]: (a) schematic diagram of the cell, (b) scanning electronmicroscopy image of $\mathrm{ZnO}$ nanowire array.

At a roughness factor of 200, the nanowire cell generates photocurrent $55-75 \%$ higher than the $\mathrm{ZnO}$ nanoparticles and comparable to the $\mathrm{TiO}_{2}$ nanoparticles $\mathrm{The} \mathrm{ZnO}$ and 
$\mathrm{TiO}_{2}$ nanowires were also emphasized to be superior to nanoparticles in electron injection kinetics. This is based on a measurement of the time constants of electron injection, indicating 3 ps for nanowires and 200 ps for nanoparticles.

\section{1. $\mathrm{TiO}_{2}$ Nanotubes}

Nanotubes are a class of very important one-dimensional nanostructure since their hollow structure may usually give surface area larger than that of nanowires or nanorods

\subsection{Bamboo-type $\mathrm{TiO}_{2}$ Nanotubes}

Unlike a constant DC potential that was most often used in literature for growth of $\mathrm{TiO}_{2}$ nanotubes, a use of $\mathrm{AV}$ potential was found to be able to produce $\mathrm{TiO}_{2}$ nanotubes with The advantage of these bamboo-type nanotubes was suggested that they presented a more porous structure and therefore might offer larger surface area for dye adsorption in comparison to those smooth-walled nanotubes created under DC condition conventionally. $\mathrm{ZnO}$ nanotubes have been also studied for application in DSCs. However, the conversion efficiencies so far achieved were generally low, $1.2-1.6 \%$. It probably results from a fact that the length of the $\mathrm{ZnO}$ nanotubes is limited by the fabrication method based on an etching mechanism.

\subsection{Zno Nanotetrapods}

$\mathrm{ZnO}$ nanotetrapods are one of typical three-dimensional nanostructures which consist of four arms extending to different spatial orientations from a common core (Fig. 9)

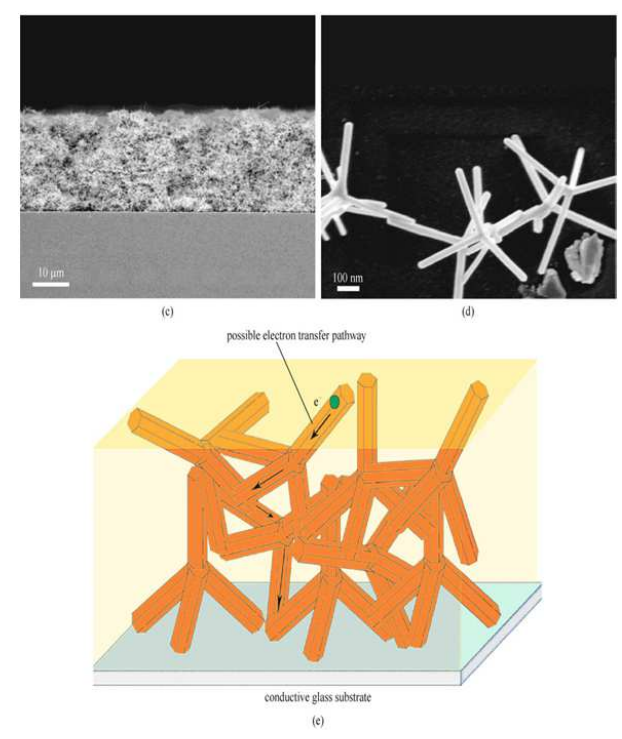

Figure 9. ZnO nanotetrapodsstructures [13]

$\mathrm{ZnO}$ nanotetrapods can be synthesized by a vapor transport deposition. The produced nanotetrapods typically feature a structure with the arms $40 \mathrm{~nm}$ in diameter and $500-800 \mathrm{~nm}$ in length. The roughness factor of a film composed of the $\mathrm{ZnO}$ nanotetrapods was demonstrated to be as high as 400 . $\mathrm{ZnO}$ nanotetrapods are of benefit to
DSCs owing to their large specific surface area in view of high roughness factor and good interconnection of the arms offering multiple pathways for electron transport.

\subsection{Branched Nanowires or Nanotubes}

Branched nanostructures are derived from onedimensional nanostructures however with a consideration to enlarge the surface area through using extended "branches', Typical branched nanostructures include (1) nanoflowers, (2) forestlike architecture, (3) branched nanowires, and (4) dendritic nanowires.

\subsection{Zno Aggregates}

Aggregates are a type of 3D nanostructure with spherical assembling of nanocrystallites or other nanomaterials. In addition to light scattering, a less compact structure of the photoelectrode film consisting of $\mathrm{ZnO}$ aggregates is also thought to be a reason giving a high conversion efficiency by the aggregate solar cells
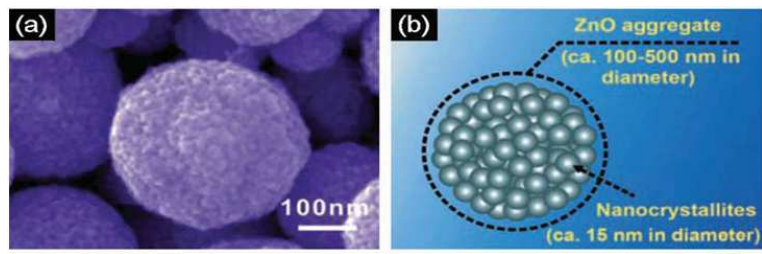

Figure 10. $\mathrm{ZnO}$ aggregates showing enhanced DSC performance in comparison with nanoparticles]: (a) SEMimage and (b) schematic drawing of $\mathrm{ZnO}$ aggregates indicating a hierarchical structure of spherical assembling of nanocrystallites [13]

Rather than a light scattering mechanism that is described in the section of $\mathrm{ZnO}$ aggregates, the high efficiency was explained by reason that the film comprised of $\mathrm{TiO}_{2}$ aggregates could provide a "highway" for electrolyte diffusion in view of the existence of $150-300$ nm-sized pores.

\section{Methods to Improve Stability and Efficiency}

1)A series of nitrogen-doped and undoped $\mathrm{TiO}_{2}$ nanocrystals was prepared by several simple methods. Needle-like N-doped $\mathrm{TiO}_{2}$ nanocrystals and nanoparticles were obtained from commercial $\mathrm{TiO}_{2}$ powders. Several dye-sensitized solar cells (DSCs) were fabricated based on $\mathrm{N}$-doped and undoped $\mathrm{TiO}_{2}$ electrodes[15]. The N-doped DSCs achieved a high conversion efficiency of $10.1 \%$ and $4.8 \%$ using an organic electrolyte and an ionic liquid electrolyte, respectively. Systemic investigations were carried out on the properties of $\mathrm{N}$-doped and undoped $\mathrm{TiO} 2$ powders, films, and DSCs. The electron transport time and electron lifetime were investigated by intensity-modulated photocurrent and photovoltage spectroscopy (IMPS/IMVS). Moreover, the electron injection of $\mathrm{N}$-doped DSCs was studied by surface photovoltage spectroscopy (SPS). The 
synergetic effect of higher dye uptake, faster electron transport and higher photovoltage contributes to a higher conversion efficiency of N-doped DSCs. The stability test also demonstrated that the photodegradation of the DSCs was not accelerated and the DSC system was stabilized by the introduction of nitrogen into the $\mathrm{TiO}_{2}$ photoelectrode. These results indicate that the $\mathrm{N}$-doped $\mathrm{TiO}_{2}$ nanocrystals prepared by our approach from commercial $\mathrm{TiO}_{2}$ are ideal semiconductor materials for DSCs.

2) Transparent and conductive carbon-based materials are promising for window electrodes in solid state optoelectronic devices. However, the catalytic activity to redox reaction limits their application as a working electrode in a liquid-type dye-sensitized solar cell (DSSC). In this letter, we propose and demonstrate a transparent carbon nanotubes (CNTs) film as the working electrode in a DSSC containing iodide/triiodide redox couples[14]. This implementation is realized by inhibiting the charge-transfer kinetics at $\mathrm{CNT} /$ redox solution interface with an aid of thin titanium oxide film that facilitates the unidirectional flow of electrons in the cell without sacrificing the electrical and optical properties of $\mathrm{CNT}$. $\mathrm{TiO}_{2}$ nanotubes and their application in dye-sensitized solar cells.

\section{Conclusions and Outlook}

DSCs have kept their efficiency record at $10-11 \%$ for many years, which are however far lower than the theoretically predicted one, $20 \%$. In principle, there are three potential ways that may achieve a breakthrough in the conversion efficiency of DSCs One way based on a consideration of boosting the light harvesting efficiency of photoelectrode is to develop new photosensitizers with higher molar extinction coefficient and broader spectral response than the existing dyes. This point has been well manifested to be both direct and effective by the history and development of DSCs over the past decades. Of course, the chemists have never stopping seeking for more powerful photosensitizers including organic dyes and other types, for example, quantum dots, and energy relay dyes . The second way is to possibly improve the open-circuit voltage of DSCs. Open-circuit voltage is known to be determined by the energy difference between the quasiFermi levels of the electrons in semiconductor and the redox couple in electrolyte. It therefore requires finding electrolytes with a redox couple more closely matched to the energy of the oxidized dye to, as such, increase the open-circuit voltage. It was estimated that a $300 \mathrm{mV}$ increase in the opencircuit voltage would mean a $35 \%$ improvement in the device efficiency, i.e., reaching $14 \%$. Some recent studies focusing on ionic liquid electrolytes show great promises in increasing the open-circuit voltage. The third way is to reduce the energy loss in the solar cell caused by, for example, charge recombination, electron trapping, optical reflection, and so on. In this regard, nanotechnology with capability of tailoring materials for defined purpose may create abundant nanostructures, and with these nanostructured materials it is possible to receive increased electron diffusion length, decreased back recombination, and physical effects such as photon localization, thus a decrease in the energy loss would likely lead to an improvement in the solar cell efficiency As for the one-dimensional nanostructures, although they have announced an extremely long electron diffusion length, up to $100 \mathrm{~m}$, currently most of the DSCs made of onedimensional nanostructures show efficiencies much lower than those obtained for the $\mathrm{TiO}_{2}$ nanoparticles. This is because of the limit in the surface area of the onedimensional nanostructures. To increase the surface area basically relies on an increase in the length of the onedimensional nanostructures.

As an outlook, the paper would like to put stress on the aggregate structure, which possesses surface area comparable to that of nanoparticles and, meanwhile, shows capability of generating light scattering. It is understood that light scattering is effective in extending the traveling distance of incident light within photoelectrode film. This may significantly enhances the light harvesting efficiency of photoelectrode.

An attempt in thorough investigation of Dye sensitized solar is made which gives a lot of information regarding structural mechanism and various nanostructures that are used to increase stability and efficiency of DSSCs.

\section{Acknowledgements}

I would like to acknowledge Prof.C.L.R.S.V. Prasad, Prinipal and Professor Nagendar Parashar Director(Academic) of GMR Institute of Technology, Rajam, A.P, India for their constant encouragement to finish this work.

\section{References}

[1] L. Kazmerski (2005), "Best Research Cell Efficiencies" [Online] [Powerpoint Presentation], accessed on March 28, 2006.

[2] O’Regan B, Gratzel M. A low-cost, high-efficiency solar-cell basedon dye-sensitized colloidal TiO2 films. Nature, 1991, 353(6346):737-740.

[3] Nazeeruddin M K, Kay A, Rodicio I, Humphry-baker R, Muller E, Liska P, Vlachopoulos N, Gratzel M. Conversion of light to electricity by Cis-X2bis(2,2'-bipyridyl-4,4'dicarboxylate)ruthenium(Ii)charge-transfer sensitizers (X = $\mathrm{Cl}-, \mathrm{Br}-, \mathrm{I}-, \mathrm{CN}-$, andSCN- ) on nanocrystalline $\mathrm{TiO}_{2}$ electrodes. Journal of the American Chemical Society, 1993, 115(14): 6382-6390.

[4] Martinson A B F, Hamann T W, Pellin M J, Hupp J T. New architectures for dye-senstized solar cells. Chemistry-A European Journal, 2008, 14(15): 4458-4467.

[5] $\mathrm{Ku} \mathrm{C} \mathrm{H}, \mathrm{Wu} \mathrm{J} \mathrm{J}$. Electron transport properties in $\mathrm{ZnO}$ nanowire array/nanoparticle composite dye-sensitized solar cells. Applied Physics Letters, 2007, 91(9): 093117. 
[6] Jiang C Y, Sun X W, Tan K W, Lo G Q, Kyaw A K K, Kwong D L. High-bendability flexible dye-sensitized solar cell with a nanoparticle-modified $\mathrm{ZnO}$-nanowire electrode. Applied Physics Letters, 2008, 92(14): 143101.

[7] Chen W, Zhang H F, Hsing I M, Yang S H. A new photoanode architecture of dye sensitized solar cell based on $\mathrm{ZnO}$ nanotetrapods with no need for calcination. Electrochemistry Communications, 2009, 11(5): 1057-1060.

[8] Yoshida T, Zhang J B, Komatsu D, Sawatani S, Minoura H, Pauporte T, Lincot D, Oekermann T, Schlettwein D, Tada H,Wohrle D, Funabiki K, Matsui M, Miura H, Yanagi H. Electrodeposition of inorganic/organic hybrid thin films. Advanced Functional Materials, 2009, 19(1): 17-43.

[9] Qiu Y C, Chen W, Yang S H. Facile hydrothermal preparation of hierarchically assembled, porous singlecrystalline $\mathrm{ZnO}$ nanoplates and their application in dyesensitized solar cells. Journal of Materials Chemistry, 2010, 20(5): 1001-1006.

[10] Chen W, Qiu Y C, Zhong Y C, Wong K S, Yang S H. HighEfficiency Dye-Sensitized Solar Cells Based on the Composite Photoanocles of $\mathrm{SnO}_{2} \quad$ Nanoparticles/ZnO Nanotetrapods. Journal of Physical Chemistry A, 2010, 114(9): 3127-3138
[11] Chen W, Qiu Y C, Yang S H. A new ZnO nanotetrapods $/ \mathrm{SnO}_{2}$ nanoparticles composite photoanode for high efficiency flexible dye-sensitized solar cells. Physical Chemistry Chemical Physics, 2010, 12(32): 9494-9501.

[12] Qiu Y C, Chen W, Yang S H. Double-layered photoanodes from variable-size anatase $\mathrm{TiO} 2$ nanospindles: a candidate for high efficiency dye-sensitized solar cells. Angewandte Chemie International Edition, 2010, 49(21): 3675-3679.

[13] Qifeng Zhang, Guozhong Cao Nanostructured photoelectrodes for dye-sensitized solar cells Nano Today (2011) 6, 91-109.

[14] A. K. K. Kyaw,1,2 H. Tantang,3 T. Wu,2 L. Ke,4 C. Peh,4 Z. H. Huang,5 X. T. Zeng,5 H. V. Demir,1,2,6 Q. Zhang,3,a) and X. W. Sun Dye-sensitized solar cell with a titaniumoxide-modified carbon nanotube transparent electrode APPLIED PHYSICS LETTERS 99, 021107 (2011).

[15] Wei Guoa, Liqiong Wua, Zhuo Chena, Gerrit Boschloob, Anders Hagfeldtb, Tingli Maa Highly efficient dye-sensitized solar cells based on nitrogen-doped titania with excellent stability Journal of Photochemistry and Photobiology A: Chemistry 219 (2011) 180-187. 